\title{
Measuring Photoexcited Free Charge Carriers in Mono- to Few-layer Transition Metal Dichalcogenides with Steady-State Microwave Conductivity
}

Jeffrey L. Blackburn, ${ }^{1 *}$ Hanyu Zhang, ${ }^{l}$ Alexis R. Myers, ${ }^{1,2}$ Jeremy Dunklin, ${ }^{l}$ David C. Coffey, ${ }^{3}$ Rebecca N. Hirsch, ${ }^{2}$ Derek Vigil-Fowler, ${ }^{1}$ Seok Joon Yun, ${ }^{4}$ Byeong Wook Cho ${ }^{4,5}$ Young Hee Lee, ${ }^{4,5}$ Elisa M. Miller, ${ }^{1}$ Garry Rumbles, ${ }^{1,6}$ Obadiah G. Reid ${ }^{1,6 *}$

1. Chemistry and Nanoscience Center, National Renewable Energy Laboratory, 15013 Denver West Parkway, Golden, Colorado 80401, USA

2. Department of Chemistry and Biochemistry, University of Colorado Boulder, Boulder, Colorado 80309, USA

3. Department of Physics, Warren Wilson College, 701 Warren Wilson Rd, Swannanoa, NC 28778, USA

4. Center for Integrated Nanostructure Physics (CINAP), Institute for Basic Science (IBS), Suwon, 16419 Republic of Korea

5. Department of Energy Science, Sungkyunkwan University, Suwon, 16419 Republic of Korea

6. Renewable and Sustainable Energy Institute, University of Colorado Boulder, Boulder, Colorado 80309, USA

\section{Supporting Information}




\section{Experimental Methods}

Sample Preparation.

Monolayer $\mathrm{WS}_{2}$ was grown on $\mathrm{SiO}_{2} / \mathrm{Si}$ wafers by atmospheric pressure chemical vapor deposition (APCVD). For synthesizing monolayer $\mathrm{WS}_{2}$, A water-soluble precursor was coated on the $\mathrm{SiO}_{2} / \mathrm{Si}$ substrate first. The precursor solution was prepared by mixing three types of chemical solutions (defined as A, B, and $\mathrm{C}$ ).

- A (Metal precursor): $0.2 \mathrm{~g}$ of ammonium metatungstate (AMT, Sigma-Aldrich, 463922), was dissolved in $10 \mathrm{~mL}$ of deionized (DI) water for tungsten precursor.

- B (Promoter): $0.1 \mathrm{~g}$ of sodium hydroxide ((NaOH, Sigma-Aldrich, 795429) dissolved in $30 \mathrm{~mL}$ DI water was introduced for promoting monolayer TMDCs.

- C (Medium solution): An OptiPrep density gradient medium (Sigma-Aldrich, D1556, 60\% (w/v) solution of iodixanol in water) was used as a medium solution.

The precursor solution in which $\mathrm{A}, \mathrm{B}$, and $\mathrm{C}$ were mixed in a certain ratios was coated onto the $\mathrm{SiO}_{2} / \mathrm{Si}$ wafer by spin-casting at $3000 \mathrm{rpm}$ for $1 \mathrm{~min}$.

The precursor-coated substrate and $0.2 \mathrm{~g}$ of sulfur (Sigma, 344621) were separately introduced to a two-zone furnace. The temperature of the sulfur zone was increased to $200{ }^{\circ} \mathrm{C}$ and $250{ }^{\circ} \mathrm{C}$ for sulfur-poor and sulfur-rich condition, respectively to modulate portion of $\mathrm{WS}_{2}$ multilayers. At the same time, the substrate zone was increased to $800{ }^{\circ} \mathrm{C}$ at a rate of $100{ }^{\circ} \mathrm{C} / \mathrm{min} .600 \mathrm{sccm}$ of Nitrogen and $10 \mathrm{sccm}$ of Hydrogen gas were injected as a carrier gas and reactive agent, respectively, to reduce metal oxides.

The as grown $\mathrm{WS}_{2}$ on $\mathrm{SiO}_{2} / \mathrm{Si}$ was transferred to quartz substrate for further optical measurement. poly(methyl methacrylate) (PMMA C4, MicroChem) was coated onto samples as a supporting layer and then immersed into diluted hydrofluoric acid for detaching $\mathrm{WS}_{2}$ from the $\mathrm{SiO}_{2} / \mathrm{Si}$ wafer by etching silicon oxide. The PMMA-supported samples were transferred to quartz substrate and then PMMA was removed by acetone.

\section{Characterization.}

\section{Confocal Photoluminescence and Raman}

Confocal PL and Raman spectra of $\mathrm{WS}_{2}$ monolayer were acquired with an InVia Renishaw confocal Raman microscope with a 532-nm laser. The laser power was measured by a Thorlabs PM100D Power Meter. For the PL measurements, the laser intensities was $260 \mathrm{~W} / \mathrm{cm}^{2}$ For the Raman measurements, the laser intensity was $1.84 \mathrm{~kW} / \mathrm{cm}^{2}$. The PL/Raman signals were dispersed by grating mirrors $(600 \mathrm{lines} / \mathrm{mm}$ grating for the PL measurements and 1800 lines/mm grating for the Raman measurements) and detected by a charge-coupled device (CCD) array.

\section{Absorption Spectroscopy}

The absorption of films was measured using a Cary 5000 optical spectrophotometer in transmission mode. The absorption is not corrected for reflection.

\section{Steady-State Microwave Conductivity}

Steady-state implementations of microwave conductivity ${ }^{46,70}$ have rarely been reported ${ }^{43}$, but provide important complimentary advantages over pulsed-laser excitation. The simplicity of measuring a steady-state microwave absorption signal allows parametric study of new variables such as excitation wavelength or applied magnetic field. In this paper we present steady-state photoconductance action 
spectroscopy; similar to measuring a photocurrent action spectrum, but with no electrical contacts. We optimized our microwave circuit for sensitive detection of square-wave modulated photoconductivity. This includes a high-quality factor microwave resonator ${ }^{46,56}(\mathrm{Q}=850)$, a low phase-noise microwave source (Rhode \& Schwartz SMB100A), a homodyne detection circuit, and a lock-in amplifier (Stanford Research, SR830). This apparatus is $\sim 100$ times more sensitive than our standard fp-TRMC apparatus. ${ }^{46}$

Figure 3 a shows a diagram of the microwave homodyne circuit and optical path. The microwave probe is split into two arms, reference and sample. The sample arm contains the sample cavity, coupled to the circuit via a circulator; the reference arm contains a variable phase shifter and attenuator. The two arms rejoin at a hybrid (magic) tee, which has a pair of matched Schottky barrier diode detectors (1N23C), one in each output arm of the tee. Their differential sensitivity is measured using a calibrated attenuator in order to quantify microwave power absorption, see SI Fig. S7. The whole circuit is implemented in WR90 (Xband) waveguide, and the isolators and circulator (M-Wave Design) all have a directivity of $\geq 35 \mathrm{~dB}$. In operation the microwave frequency $(\sim 9.9 \mathrm{GHz}, 100 \mathrm{~mW})$ is tuned to resonance with the sample cavity, the amplitude in each arm is equalized, and the relative phase is set to $90^{\circ}$. Interference of the reference and sample signals in the magic tee produces equal output power at the two detectors; any change in sample conductivity due to optical excitation manifests as an imbalance of the two detector signals, which we acquire and selectively amplify using a differential voltage amplifier (Teledyne LeCroy DA1855) and a lock-in (Stanford Research SR830).

Excitation is provided by a $150 \mathrm{~W}$ xenon arc lamp coupled to a monochromator (OBB Tunable Power Arc, $0.3 \mathrm{~m} \mathrm{f} / 4$ monochromator, $1200 \mathrm{lp} / \mathrm{mm}$ grating). The output beam is mechanically chopped (Stanford Research SR540) and sampled using an amplified photodiode to provide an excitation reference channel (acquired by a second lock-in, Stanford Research SR510). The remaining output is columnated by a $45^{\circ}$ off-axis parabolic mirror (OAP, f/4, $50 \mathrm{~mm}$ dia., Edmonds Optics) and reimaged onto an opening in the sample cavity by a second OAP (f/2, $50 \mathrm{~mm}$ dia.). The latter optical relay configuration allows the microwave circuit to be placed as far as possible from the optical chopper while also maximizing optical coupling efficiency. The former consideration is important, as one of the main noise sources in the present instrument configuration is vibrational coupling with the optical chopper. Microwave-transparent polymer shims are used to prevent the sample from vibrating within the microwave cavity. We note that, as with our TRMC measurement, the SSMC measurement reported here measures the average photoconductance of the sample, since the visible excitation source and the microwave probe beam illuminate the entire $1 \mathrm{x} 2$ $\mathrm{cm}$ quartz substrate on which the sample is deposited.

Square wave optical excitation of the sample induces a square-wave modulation in its conductivity; microwave power is absorbed, and a square-wave amplitude modulation is encoded on the microwave probe. Interference with the reference arm demodulates this signal, which appears as a voltage difference between the two microwave detectors. The lock-in amplifier detects the first Fourier series component of this signal and returns its root-mean-squared amplitude. Thus, it follows that the peak-to-peak voltage modulation of the original square-wave signal is:

$$
\Delta V_{S}=\frac{\pi \sqrt{2}}{4} V_{L I A}
$$

From this point the analysis is identical to that for fp-TRMC data. We calculate the change in microwave power reflection according to:

$$
\frac{\Delta P}{P}=n\left(V_{\text {Cell }}\right) \frac{\Delta V_{S}}{V_{\text {Cell }}}
$$

where $n\left(V_{C e l l}\right)$ is an empirical calibration function that describes the sensitivity of the detector, and $V_{\text {Cell }}$ is the equilibrium voltage output of the two detectors when the microwave frequency is on resonance with the cavity, and the interferometer is balanced. The photoconductance is calculated as:

$$
\Delta G=-\frac{1}{K} \frac{\Delta P}{P}
$$


Where $K$ is the sensitivity factor of the microwave cavity, calculated form electromagnetic simulations, which have been thoroughly described previously. ${ }^{46}$ In the present case, $K=198,000$.

The final transformation we perform is to normalize the photoconductance by the photon flux at each excitation wavelength, which is calculated from the reference diode measurement and a calibration measurement using a NIST-traceable silicon photodiode (Newport 818-UV) mounted at the sample position. However, the relationship between steady-state photoconductance and light intensity depends on the recombination order that obtains in any given sample. First order recombination leads to a steady-state charge density that is linear with light intensity, while second order recombination leads to a square-root dependence. In the present case, all the $\mathrm{WS}_{2}$ samples displayed near-perfect square-root behavior, suggesting that bimolecular recombination predominates. This is accounted for in calculating the figure of merit displayed in Figure 3b. See supporting information for details.

\section{Flash Photolysis Time-resolved Microwave Conductivity}

For TRMC measurements, the $\mathrm{WS}_{2}$ samples were pumped with a $5 \mathrm{~ns}$ pulse width beam from an optical parametric oscillator (OPO) pumped by the third harmonic of an Nd:YAG laser, and probed by microwaves at around $9 \mathrm{GHz}$. The microwave field is absorbed by photogenerated mobile carriers, and its relative change in power $\Delta \mathrm{P}$ can be measured. The change in microwave power relates to the photoconductivity $\Delta \mathrm{G}$ through $\Delta P / P=-K \Delta G$ where $K$ is an empirically determined calibration factor for the microwave cavity used in this experiment. For the TRMC measurements reported here, $K=24,000$. The cavity response time is $7 \mathrm{~ns}$. The photoconductivity is proportional to the number of charges and their mobility. It can be expressed as $\Delta G=e \beta F A I O(\phi \Sigma \mu)$ where $e$ is the elementary charge, $\beta=2.2$ is the geometric factor for the $\mathrm{X}$-band waveguide used, $I O$ is the incident photon flux, $F A$ the fraction of light absorbed at the excitation wavelength, $\phi$ is the quantum efficiency of free carrier generation per photon absorbed and $\Sigma \mu=\mu e+\mu h$ (the sum of the mobilities of electrons and holes). Biexponential fits of the photoconductivity decay transients were weighted to calculate the average carrier lifetime using the equation: $\tau$ avg $=(A 0 \tau 0+A 1 \tau 1) /(A O+A 1)$.

\section{Transient Absorption Spectroscopy}

Transient absorption spectra and dynamics were measured with an Ultrafast Systems laser system, using the "Helios" configuration, which employs pump and probe pulses with widths of ca. $200 \mathrm{fs}$ and dynamic range for the pump-probe delay of ca. $5 \mathrm{~ns}$. The system is based on a $1 \mathrm{kHz}$ regeneratively amplified Ti:sapphire laser system that produces $4 \mathrm{~mJ}$ laser pulses at $800 \mathrm{~nm}$. The Ti:sapphire laser pumps an optical parametric amplifier (OPA) to generate the $520 \mathrm{~nm}$ pump light. The excitation pulse energy employed for exciting the $\mathrm{WS}_{2}$ samples was ca. $85 \mathrm{~nJ}$ at $520 \mathrm{~nm}$, with a spot size of ca. 600 microns. A portion of the amplified $800 \mathrm{~nm}$ light was passed through a sapphire plate to generate the visible ( $400 \mathrm{~nm}<\lambda_{\text {probe }}<800 \mathrm{~nm}$ ) continuum probe pulse. The probe pulses were delayed in time with respect to the pump pulse using a motorized translation stage mounted with a retroreflecting mirror. All TA measurements were performed in an air-free optical holder to avoid sample degradation in the presence of oxygen and irradiation. 
(a)

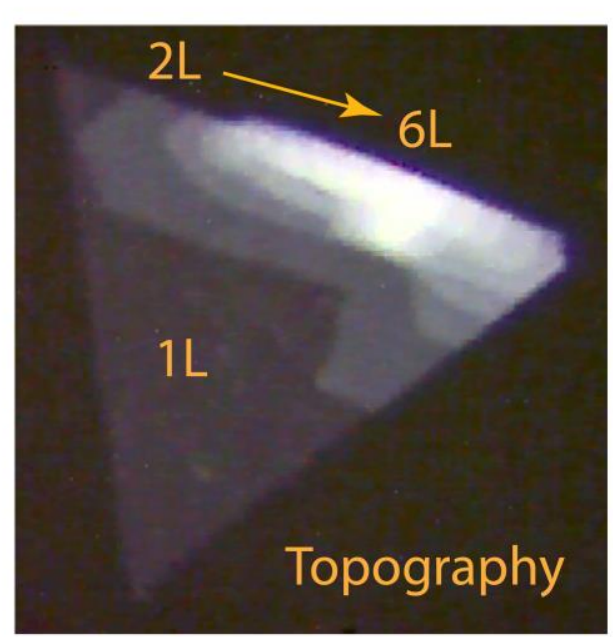

(c)

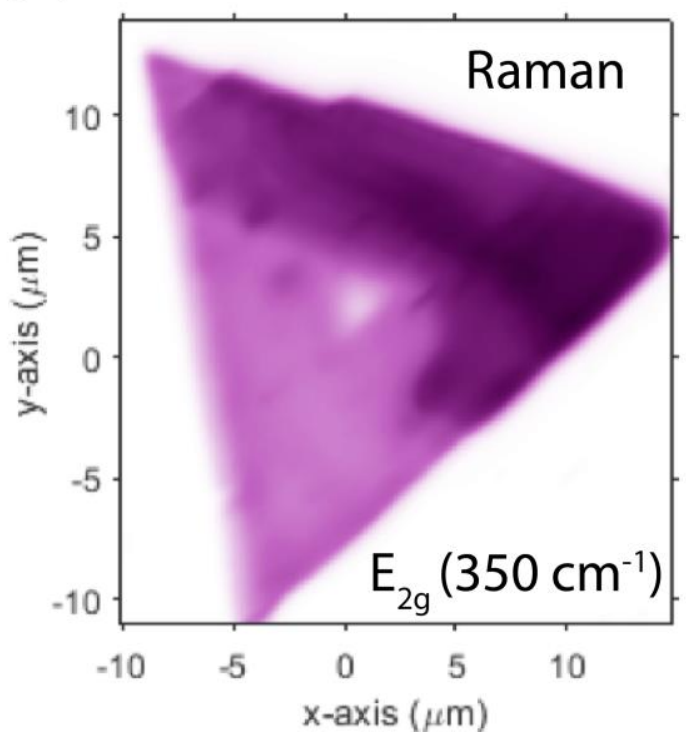

(b)

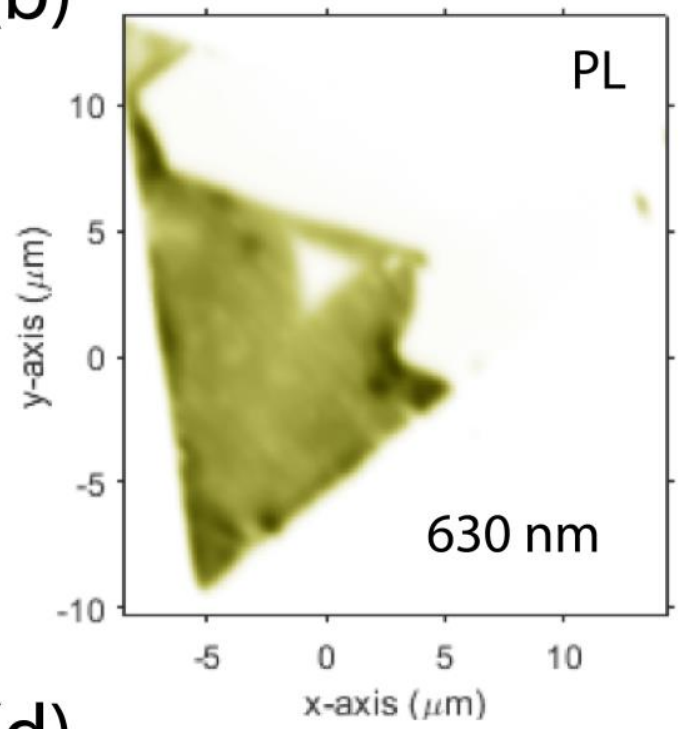

(d)

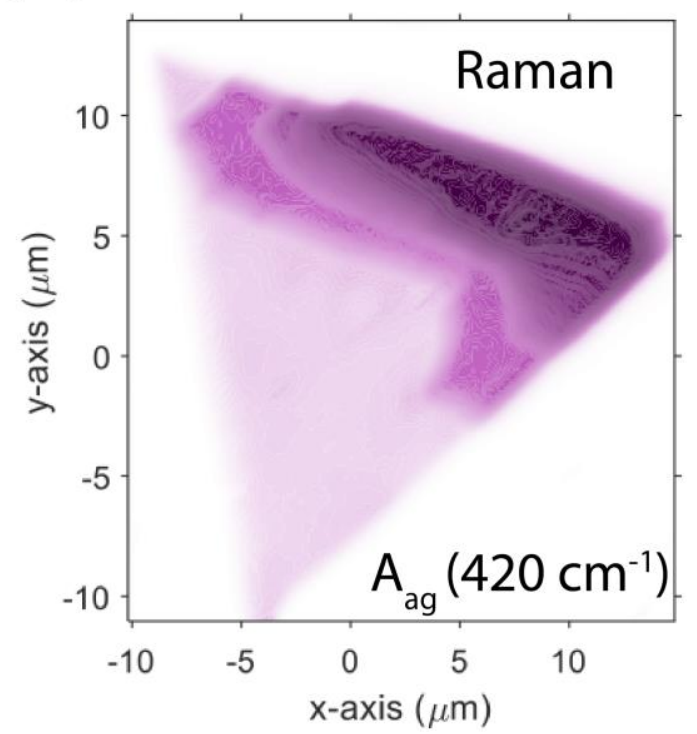

Figure S1. (a) AFM topography of a $\mathrm{WS}_{2}$ flake where six layers can be clearly discerned. (b) PL map of same flake, taken with an excitation wavelength of $532 \mathrm{~nm}$ and with an emission wavelength of $630 \mathrm{~nm}$. (c) and (d) Raman maps of same flake, taken with an excitation wavelength of $532 \mathrm{~nm}$ and for Raman shifts of $350 \mathrm{~cm}^{-1}$ $\left(\mathrm{E}_{2 \mathrm{~g}}\right.$ mode, panel c) and $420 \mathrm{~cm}^{-1}\left(\mathrm{~A}_{1 \mathrm{~g}}\right.$ mode, panel d). 
(a)

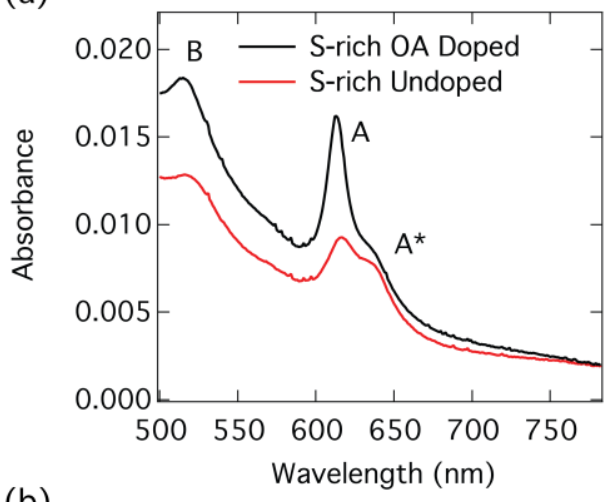

(b)

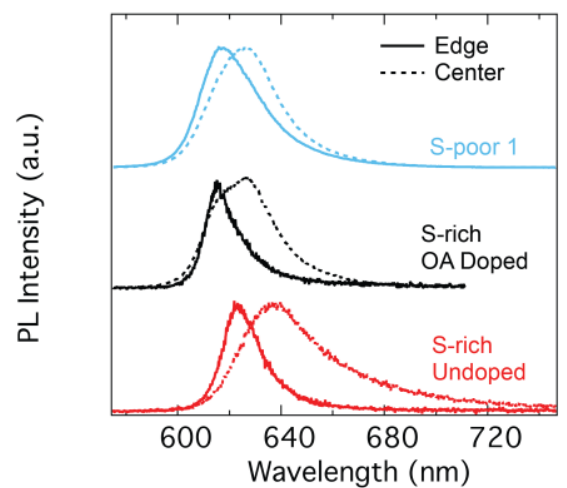

(c)

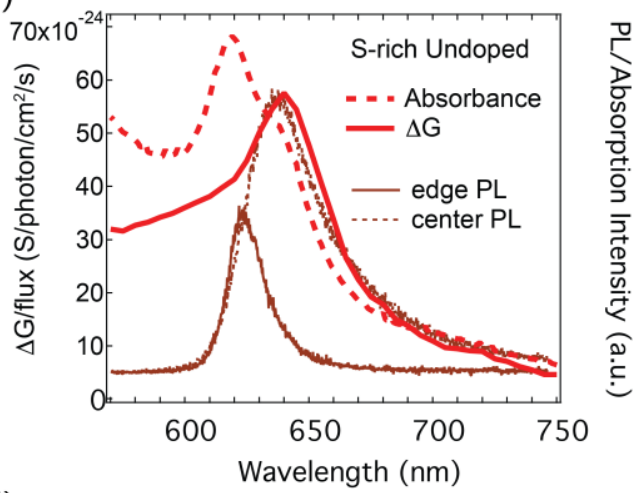

(d)

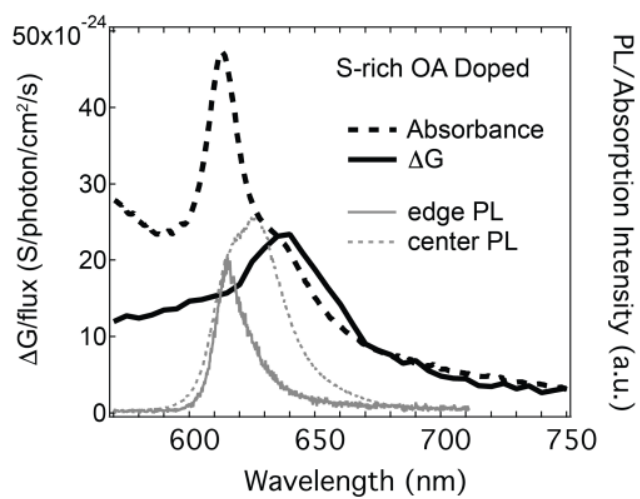

Figure S2. (a) Absorbance spectra of $\mathrm{WS}_{2}$ sample grown in sulfur-rich conditions before and after p-type doping with OA. (b) Normalized PL spectra near edge (solid lines) and center (dotted lines) of sulfur-poor sample \#1, the undoped sulfur-rich sample, and the OA-doped sulfur-rich sample. (c) Absorbance spectrum (dashed red line), photoconductance action spectrum (solid red line), PL spectrum of edge (solid auburn line) and PL spectrum of center (dotted auburn line) for undoped sulfur-rich. (d) Absorbance spectrum (dashed black line), photoconductance action spectrum (solid black line), PL spectrum of edge (solid gray line) and PL spectrum of center (dotted gray line) for OA-doped sulfur-rich sample.

\section{Analysis of Photoluminescence}

Figure S2 shows PL maps of three of the samples. PL maps of the undoped and doped $\mathrm{WS}_{2}$ monolayers revealed interesting trends for the spatial dependence of emission peak intensities and shapes. First, the PL of the $\mathrm{WS}_{2}$ flakes tended to be significantly higher along the edges than in the center of the flake, and the PL of all samples systematically shifted to the red (bathochromic) and broadened in going from the edge of the $\mathrm{WS}_{2}$ flake to the center of the flake (Fig. S2c). The emission line shape of these flakes is complex, and requires deconvolution into several peaks. We discuss this emission, and its dependence on environmental factors, excitation fluence, and temperature in detail in another manuscript that is currently under review. Most pertinent to this discussion is that the red-shifted PL can be deconvolved into peaks that most likely correspond to a combination of trion emission and what has been referred to in the literature as 'localized state' emission.

Comparison of the PL and photoconductance action spectra (Figure S2c and S2d) are particularly informative for discerning between the potential roles of trions or multi-layers in producing free charges in the photoexcited flakes. In the undoped sulfur-rich sample, the broad red-shifted PL associated with the center of the flake coincides well with both the absorption of the sub-bandgap peak and the predominant peak in the photoconductance (all occurring at ca. 630 - $635 \mathrm{~nm}$ ). Interestingly, even when this sample is compensated via p-type doping with $\mathrm{OA}$, the photoconductance action spectrum is dominated by the same peak at $635 \mathrm{~nm}$. As discussed in the manuscript, and shown again in Figure S2a, the A* absorbance shoulder at $630 \mathrm{~nm}$ does not change upon OA doping. Remarkably, however, the red-shifted PL (which has a large 
contribution from trion emission) shifts to the blue and narrows significantly upon OA doping. Importantly, the red-shifted emission of the OA-doped sample $(625 \mathrm{~nm})$, which we believe arises primarily from trion emission, is both more narrow and significantly higher in energy than both the $\mathrm{A}^{*}$ absorbance peak and the peak of the photoconductance, suggesting that the free charge carriers measured in the microwave conductivity experiment do not result predominantly from direct optical excitation of trions.

\section{(a) S-poor 1 Total Coverage}

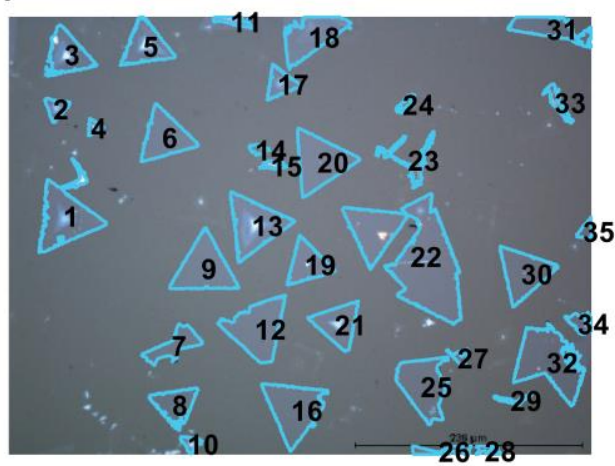

(b)

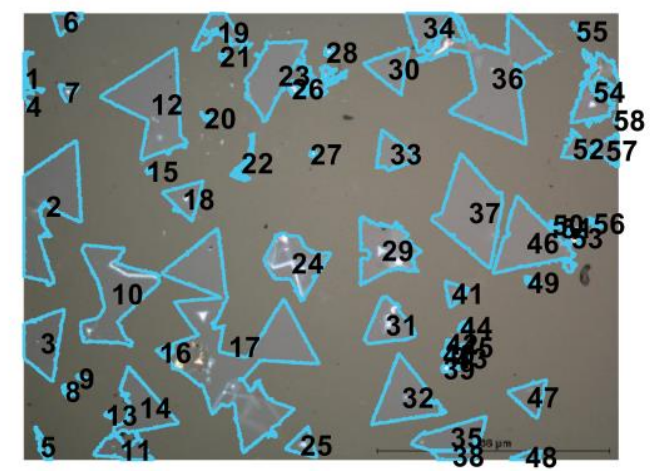

(c)

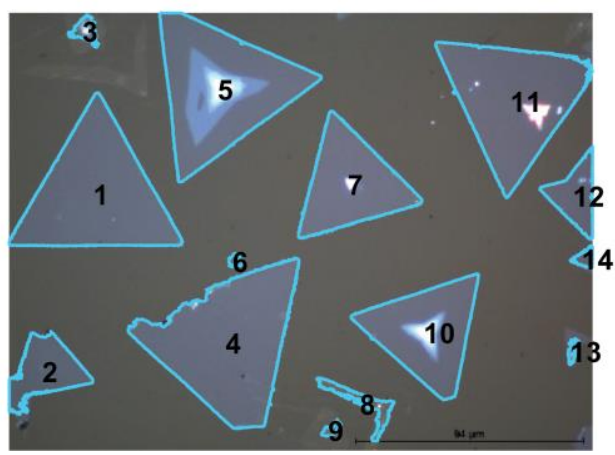

\section{S-poor 1 Multilayer Coverage}
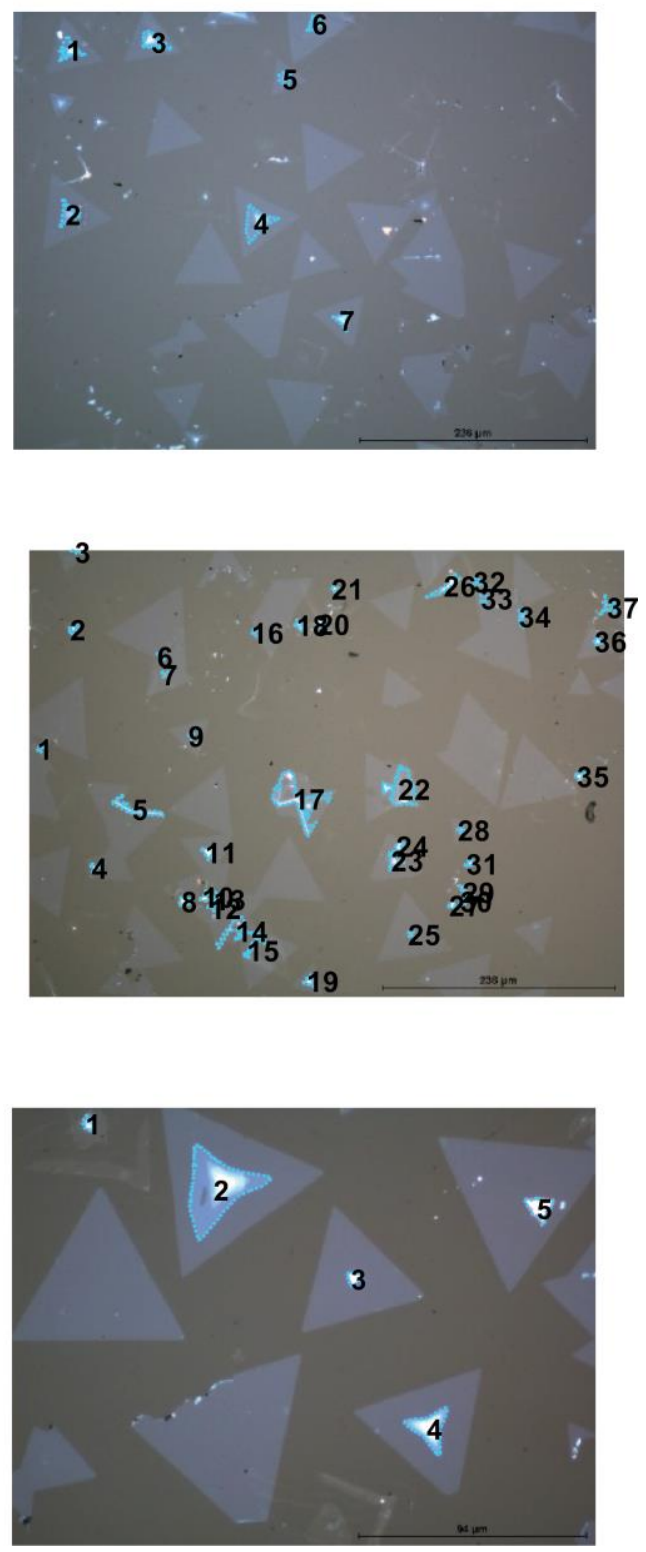

Figure S3. Microscope image analysis of the S-poor 1 sample to determine the ratio between the area covered by multilayers (right panels) to the total area covered by $\mathrm{WS}_{2}$ (left panels). Panels a, $\mathrm{b}$, and $\mathrm{c}$ represent three different areas of the sample. After imaging a large number of flakes, the ratio of the multilayer area to the total area for this sample was estimated to be $8.8 \%(+/-3.6 \%)$. 


\section{Discussion of Image Analysis Algorithm}

In the current study, we utilize a limited number of images from several random spots on a given substrate to provide an approximation of the overall, monolayer, and multilayer coverages. We consider the following points with respect to the statistical uncertainties associated with this algorithm and the potential for "false positive" identification of mono- or multi-layer sections. If "false positive" refers to identified objects (i.e., the incorrect discrimination between mono/multilayer), this is fairly trivial to avoid in our current scenario. Here, we adjust the image contrast distribution and manually set the selection threshold of the objects for each image. Using this procedure, we can identify essentially no false positives or false negatives. However, if this procedure were applied to a very large number of images where the contrast varied significantly from image to image, we could envision that some machine learning may need to be incorporated into the algorithm to appropriately auto-adjust the selection threshold for each image when appropriate. The calculated percentage of mono- and multilayers, in terms of the segmentation of pixels at the mono/multilayer interface, also relies on the acutance of an object (i.e. how sharply the contrast changes in going from mono- to multi-layer). In an attempt to estimate the standard deviation arising from this pixel segmentation uncertainty, we ran the algorithm with subtly varying selection thresholds. In this control experiment, the coverages extracted for each component change less than $2 \%$. The number is appreciably less than the statistical variations of the mono/multilayer distribution (i.e., $+/-4-13 \%$ ) that arise from image to image (i.e. by imaging many different random areas of the substrate). These control experiments point to the ranges considered statistically significant for our estimation.

(a)

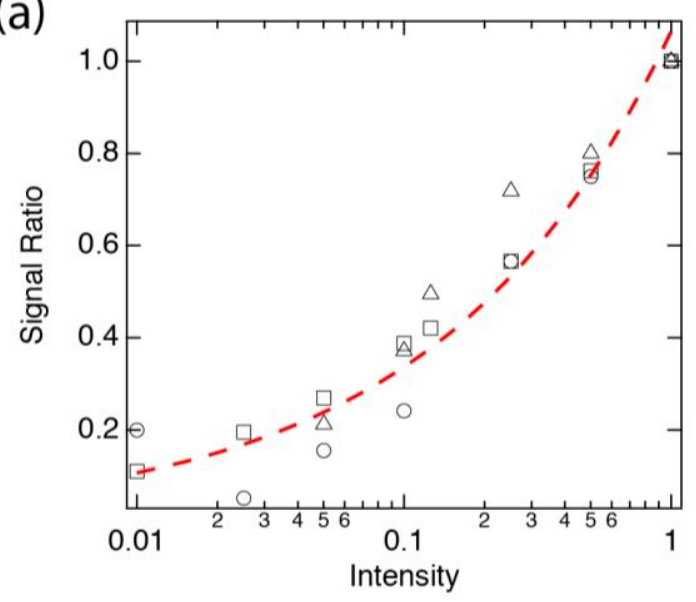

(b)

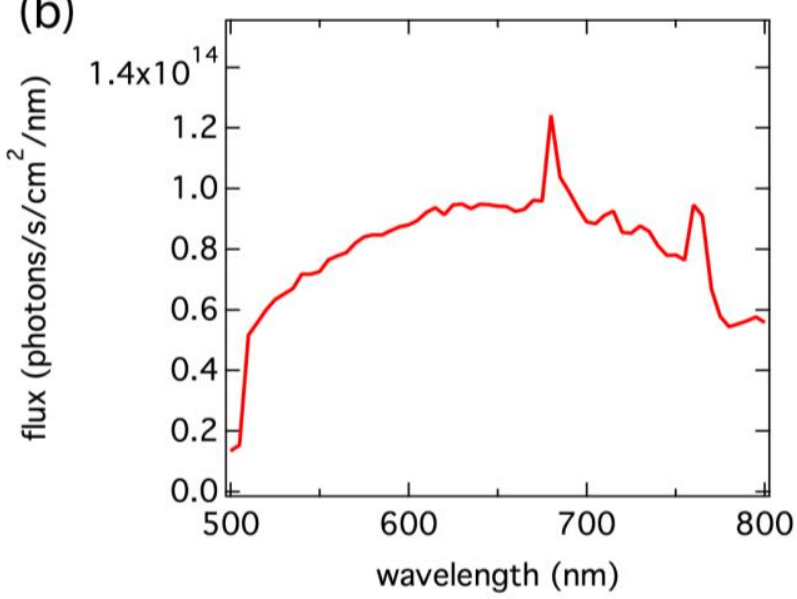

Figure S4. (a) Normalized steady-state microwave conductivity signal as a function of relative light intensity (markers) for all three $\mathrm{WS}_{2}$ samples presented in the main text. The dashed line shows a square-root dependence of the signal on light intensity. (b) Typical spectrum of the xenon arc lamp used in SSMC experiments, giving the spectral irradiance at the sample position a $490 \mathrm{~nm}$ long pass filter causes the irradiance to drop rapidly toward $500 \mathrm{~nm}$, and prevents $2^{\text {nd }}$ order diffracted light from contributing erroneously to the microwave conductivity spectrum. 

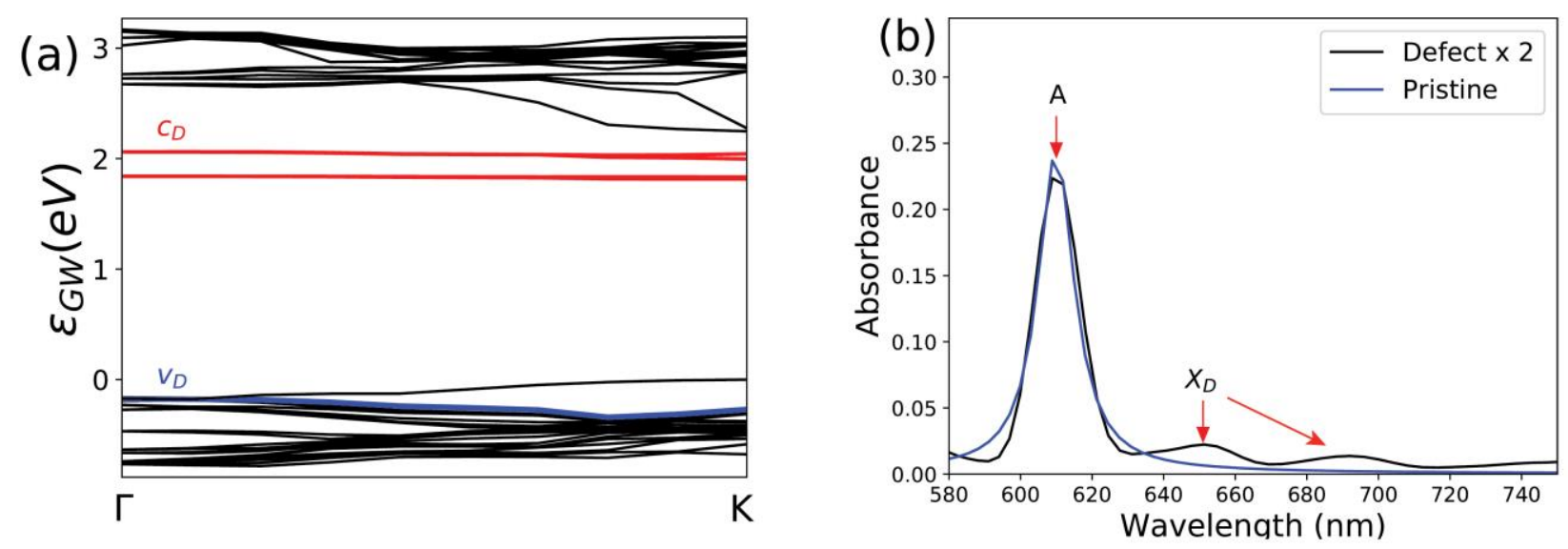

Figure S5. (a) Computed band structure from $\Gamma$ point to $K$ point. There are 4 unoccupied defect states in the gap, and one occupied defect state in the valence-band manifold. (b) Theoretically computed absorption spectrum of WS $\mathrm{W}_{2}$ with basal plane sulfur-vacancy defect (black) and pristine $\mathrm{WS}_{2}$ (blue). Defect-related peaks arise at $650 \mathrm{~nm}, 690 \mathrm{~nm}(\mathrm{shown}$ above), as well as $750 \mathrm{~nm}, 775 \mathrm{~nm}, 850 \mathrm{~nm}$, and $930 \mathrm{~nm}$ (not shown).

\section{Theoretical Methods}

To generate wavefunctions and mean-field energies needed for subsequent GW and GWBSE calculations, we performed density functional theory calculations using the Quantum ESPRESSO package. ${ }^{71,72} \mathrm{We}$ used the fully-relativistic, two-component formalism due to the presence of tungsten and used the fully relativistic pseudopotentials from the SG15 library, ${ }^{73-75}$ which employs the PBE functional. ${ }^{76}$ Following Qiu and Refaely, ${ }^{77}$ our simulation cell is a 5x5x1 supercell of $\mathrm{WS}_{2}$ with one sulfur atom removed, since this is the smallest super cell that does not give spurious interactions between the periodic images of the defects when using periodic boundary conditions. We fixed the lattice constant at the experimental value and relaxed the atomic positions after removing the sulfur atom. Our wavefunction cutoff is 60 Ry and reciprocal space sampling is $4 \times 4 \times 1$ for the charge density, and $3 \times 3 \times 1$ for the wavefunctions used for the $\mathrm{GW}$ calculations.

For our GW and GW-BSE calculations, we use the fully-relativistic, two-component formalism implemented in the BerkeleyGW package ${ }^{78-80}$ to get the most accurate results possible. For the GW calculations, we use a 15 Ry cutoff and 10,000 bands in our perturbation theory summations, model the frequency dependence of the dielectric response using the HybertsenLouie generalized plasmon-pole model, ${ }^{78-80}$ and speed convergence by using the static COHSEX correction. ${ }^{81} \mathrm{We}$ exclude the states coming from the tungsten $4 \mathrm{f}$ orbitals from our summations in calculating the dielectric matrix since they are $30 \mathrm{eV}$ below the VBM and do not change our results. For the GW-BSE calculations, we use the interpolation scheme in the BerkeleyGW package to solve the BSE with a reciprocal space sampling of $15 \times 15 \times 1$. We use the Tamm-Dancoff approximation and include 20 valence and conduction states to compute the absorption spectrum, and 40 valence and conduction states on the coarse grid needed for the interpolation scheme. This converges the spectra in the range show in figure 4 . The theoretical spectra for defected $\mathrm{WS}_{2}$ are red shifted by $0.2 \mathrm{eV}(50 \mathrm{~nm})$ due to an underestimation of the band gap when including relativistic effects with SG15 pseudpotentials, ${ }^{73-75}$ so we shift our spectra to match the location of the A peak in the experimental data. A similar shifting is also done for the pristine $\mathrm{WS}_{2}$ data for comparison. 

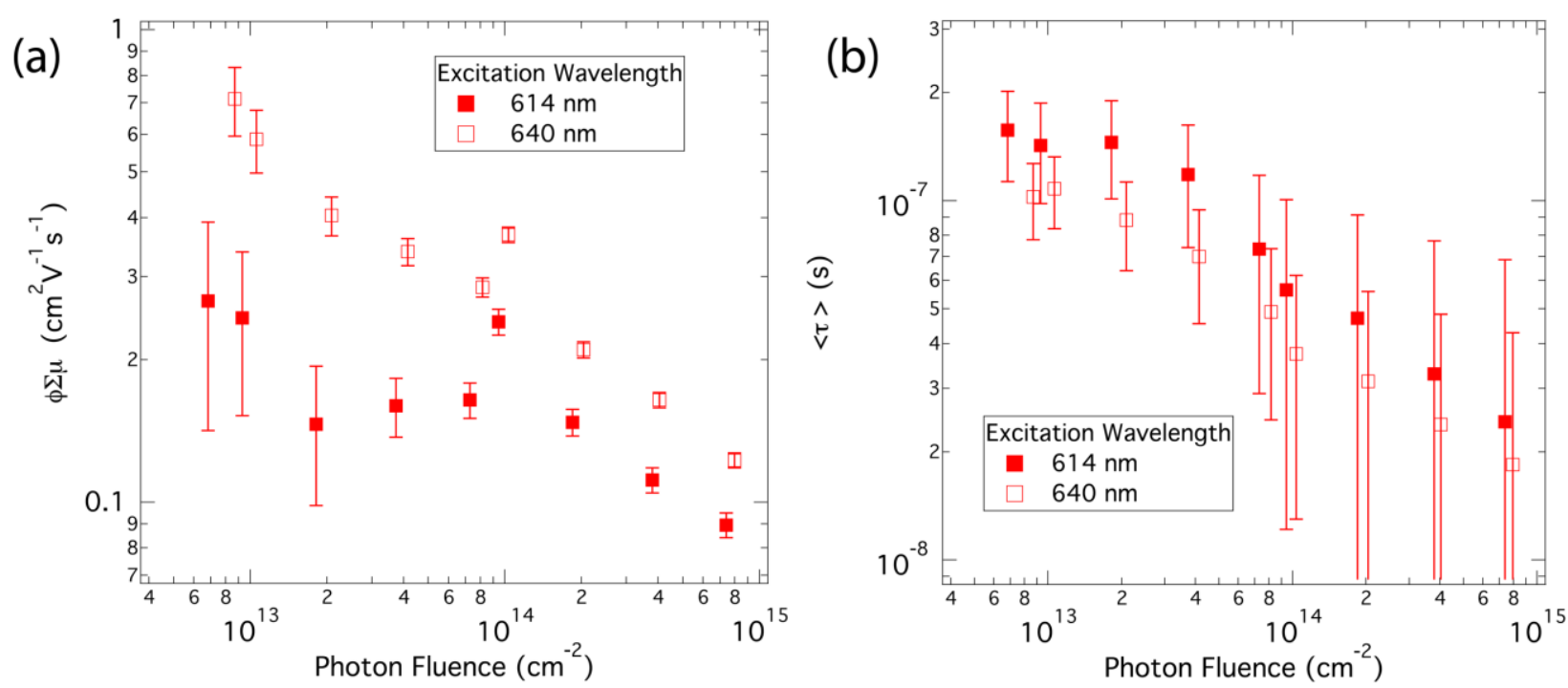

Figure S6. Time-resolved microwave conductivity results for S-rich $\mathrm{WS}_{2}$ sample, either excited at the A exciton (614 $\mathrm{nm}$ ) or the $\mathrm{A}^{*}$ exciton $(640 \mathrm{~nm})$. (a) Fluence dependence of the yield-mobility product. (b) Fluence dependence of the average photoconductance lifetime. The decrease in charge yield observed in panel (a) is most likely due to excitonexciton annihilation and is not thought to be connected with the sublinear flux-dependence of the SSMC signal shown in figure S4.

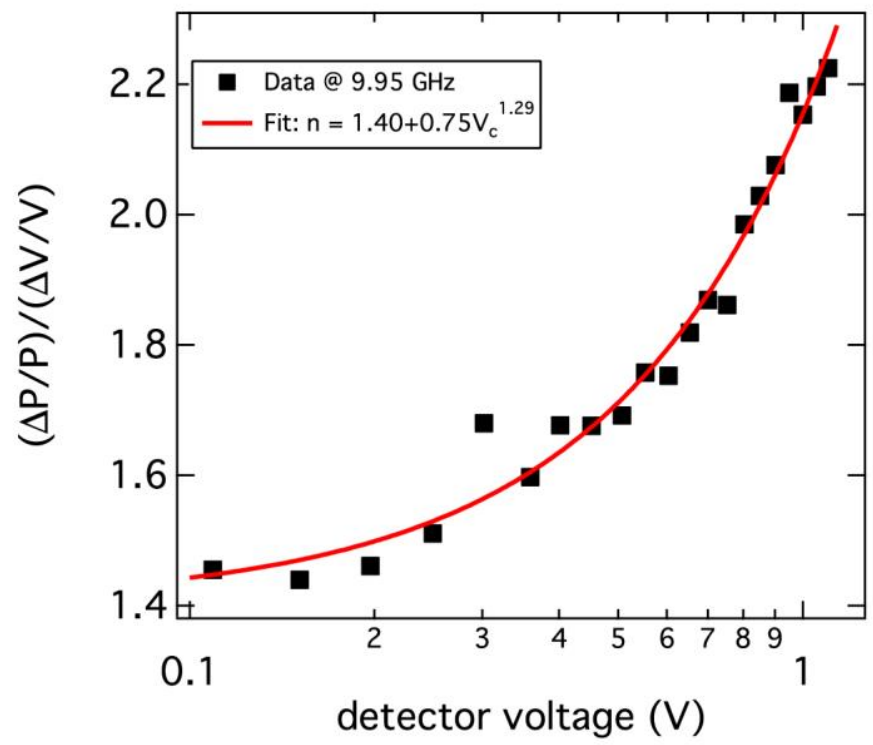

Figure S7. Measured gradient of detector voltage with respect to microwave power (black squares). The "nfunction" used to convert transient change in detector voltage to microwave power is given by the power law fit (red solid line). 


\section{Calculating the figure of merit for SSMC data:}

Steady-state and transient conductivity are fundamentally different quantities. The latter is derived from a nearly instantaneously generated population of charges, with explicitly recorded recombination processes. The latter implicitly includes both recombination and generation terms, as steady state is only reached when the rates of generation and that of recombination are equal. Two main situations are common in semiconductors: through trap recombination that results in $1^{\text {st }}$ order kinetics, and bimolecular recombination that results in $2^{\text {nd }}$ order kinetics. These are defined by the equations:

$$
\begin{aligned}
& \frac{d[n](t)}{d t}=g-k_{r}[n] \\
& \frac{d[n](t)}{d t}=g-\gamma_{r}[n]^{2}
\end{aligned}
$$

Where $[n]$ is the concentration of charges, $g$ is the rate at which they are generated, $k_{r}$ is the first-order recombination rate constant, and $\gamma_{r}$ is the bimolecular recombination rate constant. In the limit as $t \rightarrow \infty$ these equations have the respective solutions:

$$
\begin{aligned}
& {[n]=\frac{g}{k_{r}}} \\
& {[n]=\sqrt{\frac{g}{\gamma_{r}}}}
\end{aligned}
$$

We wish to define a figure of merit in each case that is proportional the yield, mobility, and lifetime of the photogenerated charges. We begin by noting that conductivity as defined as:

$$
\sigma=q[n]\left(\mu_{e}+\mu_{h}\right)=q[n] \Sigma \mu
$$

Where $\mu$ is the charge carrier mobility, and $q$ is the elementary charge. $g$ can be expanded as:

$$
g=\frac{\varphi I_{0} F_{A}}{d}
$$

Where $\varphi$ is the charge carrier yield per absorbed photon, $I_{0}$ is the incident photon flux, $F_{A}$ is the fraction of light absorbed by the sample, and $d$ is the sample thickness. The quantity we measure in or SSMC experiment is the steady state photoconductance, $\Delta G$, which can also be expanded in terms of the conductivity and the geometry of the sample:

$$
\Delta G=\Delta \sigma \frac{b d}{a}
$$

Where $b$ is the long dimension of the waveguide cross section, and $a$ is the short dimension of the waveguide crosssection.

Substituting (3), (5), and (6) into (7) and re-arranging gives the desired figure of merit for a system in which firstorder recombination dominates:

$$
\frac{\varphi \Sigma \mu}{k_{r}}=\frac{\Delta G a}{I_{0} F_{A} q b}
$$

And doing the same using equation (4) gives that for a system where bimolecular recombination dominates:

$$
\frac{\varphi(\Sigma \mu)^{2}}{\gamma_{r}}=\frac{\Delta G^{2} a^{2}}{I_{0} F_{A} q^{2} b^{2} d}
$$

As noted in the main text and shown in Figure S4a, the present samples exhibit predominantly bimolecular recombination, and equation (9) is used to calculate the SSMC spectra displayed in Figure 3b. It is also notable that 
the sample thickness does not cancel out of equation 9 as it does in equation 8 . For our calculations we used a nominal value of $1 \mathrm{~nm}$, as being approximate to few layer areas of the tungsten disulfide film. As this is only a global scaling factor it does not affect any of our conclusions, which hinge upon the shape of the SSMC spectrum. 